\title{
Correlations in the Initial Conditions of Heavy-Ion Colli- sions
}

\author{
Douglas Wertepny ${ }^{1, *}$, Jacquelyn Noronha-Hostler ${ }^{2, * *}$, Matthew Sievert ${ }^{2, * * *}$, Skandaprasad \\ $\mathrm{Rao}^{3, * * * *}$, and Noah Paladino ${ }^{3, \dagger}$ \\ ${ }^{1}$ Department of Physics, Ben-Gurion University of the Negev, Beer-Sheva 84105, Israel \\ ${ }^{2}$ University of Illinois at Urbana-Champaign, Urbana, IL 61801, US \\ ${ }^{3}$ Department of Physics and Astronomy, Rutgers University, Piscataway, NJ USA 08854
}

\begin{abstract}
.
Ultracentral collisions of heavy nuclei, in which the impact parameter is nearly zero, are especially sensitive to the details of the initial state model and the microscopic mechanism for collective flow. In a hydrodynamic "flow" picture, the final state momentum correlations are a direct response to the fluctuating initial geometry, although models of the initial geometry differ widely. Alternatively, dynamical mechanisms based in the color glass condensate (CGC) formalism can naturally lead to many-body correlations with very different systematics. Here we present a calculation of event-by-event elliptic flow in both the hydrodynamic and CGC paradigms and show that they can be qualitatively distinguished in ultracentral collisions of deformed nuclei. Specifically, the multiplicity dependence in such collisions is qualitatively opposite, with the CGC correlations increasing with multiplicity while the hydrodynamic correlations decrease. The consistency of the latter with experimental data on $U U$ collisions appears to rule out a CGC-mediated explanation. We find that these qualitative features also persist in small deformed systems and can therefore be a valuable test of the microscopic physics in that regime.
\end{abstract}

\section{Introduction}

This Proceedings is a brief summary of the important results derived in Ref. [1]; we refer the interested reader there for additional information and details.

While "centrality" in heavy-ion collisions is usually considered to be synonymous with "impact parameter," the physics which drives multiplicity production arises from many factors. In off-central collisions (say, centralities greater than $\sim 10 \%$ ), both the produced multiplicity and the elliptical collision geometry are indeed strongly correlated with the impact parameter $b$. But in ultracentral collisions (say, centralities less than $\sim 1 \%$ ), where $b \approx 0$ already, the remaining variation in multiplicity production is sensitive to the presence of deformed nuclear geometry. For this reason, ultracentral collisions of deformed ions such as

\footnotetext{
*e-mail: wertepny@ post.bgu.ac.il

**e-mail: jnorhos@illinois.edu

***e-mail: msievert@illinois.edu

****e-mail: svr34@scarletmail.rutgers.edu

†e-mail: noah.paladino@rutgers.edu
} 
uranium have been used to study the impact of nonspherical nuclear geometries [2-4]. This kind of geometry engineering in ultracentral collisions has been used to rule out the twocomponent Glauber model of initial conditions [5-7] and also extended to other deformed nuclei such as xenon [8-11]. As we demonstrate in this work, such ultracentral collisions of deformed nuclei can also provide a powerful test of the response of the system to initial geometry in various theoretical frameworks. Specifically, we find that two competing models for the microscopic origin of collective flow - hydrodynamics and the color glass condensate - lead to qualitatively opposite predictions for the multiplicity dependence in ultracentral collisions of deformed systems. This is especially important for understanding the role which non-hydrodynamic response may play in small deformed systems $[12,13]$ and how the transition from large systems with hydrodynamic response may occur [14].

In distinguishing collective flow in hydrodynamics from other multiparticle correlations, it is useful to decompose the two-particle distribution for a given event into "factorized" and "non-factorized" pieces [15]:

$$
\frac{d N_{2}}{d^{2} p_{1} d y_{1} d^{2} p_{2} d y_{2}}=\frac{d N_{1}}{d^{2} p_{1} d y_{1}} \frac{d N_{1}}{d^{2} p_{2} d y_{2}}+\delta_{2}\left(p_{1}, p_{2}\right) .
$$

Here the first term factorizes into two particles produced independently, and the second term denotes the intrinsic, non-factorizing correlations. From here one can define the second cumulant $v_{n}\{2\}$ as

$$
\left(v_{n}\{2\}\right)^{2} \equiv \frac{\left\langle\int_{p_{1} p_{2}} e^{i n\left(\phi_{1}-\phi_{2}\right)} \frac{d N_{2}}{d^{2} p_{1} d y_{1} d^{2} p_{2} d y_{2}}\right\rangle}{\left\langle N_{\text {pairs }}\right\rangle} .=\frac{\left\langle\left|\int_{p} e^{i n \phi} \frac{d N_{1}}{d^{2} p d y}\right|^{2}\right\rangle}{\left\langle N_{\text {pairs }}\right\rangle}+\frac{\left\langle\int_{p_{1} p_{2}} e^{i n\left(\phi_{1}-\phi_{2}\right)} \delta_{2}\left(p_{1}, p_{2}\right)\right\rangle}{\left\langle N_{\text {pairs }}\right\rangle} .
$$

The first term here reflects the indirect correlation of two particles because of their mutual correlation with the event geometry; this is the phenomenon of "collective flow" in hydrodynamic response. The second term represents the dynamical "non-flow" effects which correlate two particles directly with each other. Usually non-flow effects such as jet correlations are suppressed through the use of rapidity cuts, although non-flow correlations which are long-range in rapidity can survive such a procedure.

\section{Collective Flow in the Hydrodynamics}

In the hydrodynamic paradigm, non-flow effects are suppressed such that all particles are generated independently. In this scenario, $\delta_{2}=0$ and all multiparticle correlations such as $v_{n}\{2\}$ arise from moments (cumulants) of the event-by-event distribution of the single-particle anisotropy

$$
V_{n} \equiv v_{n} e^{i n \psi_{n}}=\frac{1}{N_{\mathrm{tot}}} \int_{p} e^{i n \phi} \frac{d N_{1}}{d^{2} p d y},
$$

such that the second-order cumulant

$$
v_{n}\{2\} \stackrel{\text { flow }}{=} \sqrt{\left\langle v_{n}^{2}\right\rangle}
$$

measures the RMS of the fluctuating anisotropic flow $v_{n}$. In turn, the flow vector $V_{n}$ for a given event arises from the deterministic response of hydrodynamics to the initial conditions. From symmetry considerations and the assumption that long-wavelength modes dominate in 
hydrodynamics, one can construct a hierarchy of estimators to $V_{n}$ from the initial state [16]. These estimators are known as eccentricities, defined as

$$
\mathcal{E}_{n} \equiv \varepsilon_{n} e^{i n \Phi_{n}}=-\frac{\left\langle\int_{r} r^{n} e^{i n \phi} s(r, \phi)\right\rangle}{\left\langle\int_{r} r^{n} s(r, \phi)\right\rangle}
$$

where here the entropy density $s$ is used to characterize the initial state. In central heavy-ion collisions, these estimators are precise enough that simple linear response

$$
\begin{gathered}
V_{n} \approx \kappa_{n} \mathcal{E}_{n} \\
v_{n}\{2\} \approx\left|\kappa_{n}\right| \sqrt{\left\langle\varepsilon_{n}^{2}\right\rangle} .
\end{gathered}
$$

provides a good description of the observed flow harmonics [16].

Within this framework, we can estimate the cumulants $v_{n}\{2\}$ in ultracentral collisions of deformed nuclei. We generate an initial collision geometry using the Trento code [17], which combines the nuclear profiles of the colliding nuclei into a reduced thickness function $T_{R}$. This specifies the initial entropy density $s(x, y) \propto T_{R}(x, y)$, from which we can determine the event-by-event eccentricities. Then linear response determines the cumulant for sub-bin $i$ relative to the full $0-1 \%$ centrality bin as

$$
\frac{v_{n}^{i}\{2\}}{v_{n}\{2\}}=\sqrt{\frac{\left\langle\varepsilon_{n}^{2}\right\rangle_{i}}{\left\langle\varepsilon_{n}^{2}\right\rangle_{0-1 \%}}} .
$$

\section{Non-Flow Gluon Correlations in the CGC}

The most prominent alternative to a hydrodynamic paradigm in certain heavy-ion collisions is an explanation based on multi-gluon correlations arising from the color-glass condensate (CGC) effective theory of high-energy QCD. Because the single-gluon distribution is isotropic,

$$
\frac{d N_{1}}{d^{2} p d y}=\frac{1}{2 \pi p_{T}} \frac{d N_{1}}{d p_{T} d y}
$$

the "collective flow" component of the second-order cumulant $v_{n}\{2\}$ vanishes. Instead, the cumulant is driven by the explicit two-gluon correlations $\delta_{2}$, which can be calculated orderby-order in the CGC framework. In the high- $p_{T}$ "dilute-dilute" limit, the gluon correlation function has an explicit dependence on the the overlapping nuclear profiles $T_{A}, T_{B}$. At leading order, that dependence is quadratic

$$
\delta_{2}\left(p_{1}, p_{2}\right) \stackrel{\text { L.O. }}{=}\left(\int d^{2} x_{\perp} T_{A}^{2}\left(\vec{x}_{\perp}\right) T_{B}^{2}\left(\vec{x}_{\perp}\right)\right) f\left(p_{1}, p_{2}\right),
$$

but the resulting gluon correlations are explicitly symmetric: $f\left(p_{1}, p_{2}\right)=f\left(p_{2}, p_{1}\right)[18,19]$. As a result, the quadratic dependence on the nuclear profiles generates the leading contribution to even harmonics $n$ such as $v_{2}\{2\}$, while the contribution to odd harmonics such as $v_{3}\{2\}$ vanishes. For these odd harmonics, the leading contribution arises at the next-to-leading order:

$$
\delta_{2}\left(p_{1}, p_{1}\right) \stackrel{\text { N.L.O. }}{=}\left(\int d^{2} x_{\perp} T_{A}^{3}\left(\vec{x}_{\perp}\right) T_{B}^{3}\left(\vec{x}_{\perp}\right)\right) g\left(p_{1}, p_{2}\right),
$$



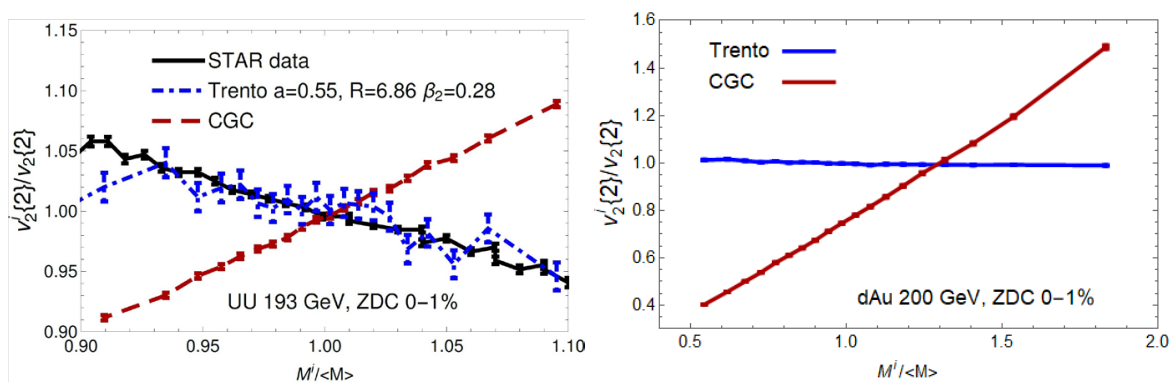

Figure 1. Comparison of the fractional elliptic flow $v_{2}\{2\}$ fractional versus multiplicity $M$ in ultracentral collisions of deformed ions. Here we show both a large deformed system (UU, left panel) and a small deformed system (dAu, right panel) and normalize the axes by the full $0-1 \%$ centrality bin.

where the symmetry is explicitly broken: $g\left(p_{1}, p_{2}\right) \neq g\left(p_{2}, p_{1}\right)$ [20]. Here the functions $f$ and $g$ stand for specific integrals of the transverse momenta.

The angular integrals which determine the second cumulant $v_{n}\{2\}$ then yield

$$
\left(v_{n}\{2\}\right)^{2}=\frac{1}{\left\langle N_{\text {pairs }}\right\rangle} \begin{cases}f_{n}\left\langle\int d^{2} x_{\perp} T_{A}^{2}\left(\vec{x}_{\perp}\right) T_{B}^{2}\left(\vec{x}_{\perp}\right)\right\rangle & \text { if } \mathrm{n}=\text { even } \\ g_{n}\left\langle\int d^{2} x_{\perp} T_{A}^{3}\left(\vec{x}_{\perp}\right) T_{B}^{3}\left(\vec{x}_{\perp}\right)\right\rangle & \text { if } \mathrm{n}=\text { odd }\end{cases}
$$

where the coefficients $f_{n}, g_{n}$ are pure numbers which will cancel out in ratios. This allows the cumulants to be calculated, event-by-event, directly from overlap integrals of the nuclear profiles

$$
\mathcal{I}_{\alpha} \equiv \int d^{2} x_{\perp} T_{A}^{\alpha}\left(\vec{x}_{\perp}\right) T_{B}^{\alpha}\left(\vec{x}_{\perp}\right)
$$

This includes not only the numerators of the cumulants which invoke $I_{2}, I_{3}$, but also the denominators $\left\langle N_{\text {pairs }}\right\rangle \propto\left\langle\left(\mathcal{I}_{0}\right)^{2}\right\rangle$ using the phenomenologically-preferred $p=0$ setting of Trento [21]. Altogether this gives for the CGC scenario

$$
\frac{v_{n}^{i}\{2\}}{v_{n}\{2\}}=\sqrt{\frac{\left\langle\left(\mathcal{I}_{0}\right)^{2}\right\rangle_{0-1 \%}}{\left\langle\left(\mathcal{I}_{0}\right)^{2}\right\rangle_{i}} \frac{\left\langle\mathcal{I}_{2,3}\right\rangle_{i}}{\left\langle\mathcal{I}_{2,3}\right\rangle_{0-1 \%}}}
$$

where one uses $I_{2}$ for $n=$ even and $I_{3}$ for $n=$ odd. In this way, we can generate an event-byevent comparison between the cumulants $v_{n}\{2\}$ arising from collective flow in hydrodynamic response and the dynamical gluon correlations arising in the CGC.

\section{Results and Conclusions}

Results for the second elliptic cumulants $v_{2}\{2\}$ are shown in Fig. 1 for both a large UU collision and a small dAu collision. In $U U$ collisions, the experimental data (black) reveal an anticorrelation between the elliptic flow and the event multiplicity. This decreasing trend is consistent with the expectation of a linear response from hydrodynamics to the initial state (blue). In this scenario, tip-on-tip collisions produce the most multiplicity but the least ellipticity, while side-on-side collisions produce less multiplicity but a greater ellipticity.

These features are qualitatively opposite to the trend shown by the CGC-mediated correlations (red), which increases monotonically as a function of multiplicity. This is because the 
particle correlations are all proportional to powers of the saturation momentum $Q_{s}^{2}$ which is monotonically correlated with multiplicity. The CGC-mediated mechanism cares only about density, not about geometry. For this reason, the predictions in ultracentral deformed systems are qualitatively opposite.

These features persist generically in smaller systems, as we discuss in detail in Ref. [1]. As an example, we also show the equivalent results for ultracentral $d A u$ collisions. The same qualitative trends persist: the hydrodynamic response is anticorrelated with multiplicity, while the CGC response is positively correlated with it. For $d A u$ the ellipticity in ultracentral collisions is not as great as in $U U$, so the negative slope persists. Meanwhile, the CGC response is still strongly correlated with multiplicity, even in this small system. Although ample $d A u$ data exists on tape, this analysis of ultracentral events has not yet been performed. We further believe that these results are generic and applicable across the entire spectrum of small and large systems.

\section{References}

[1] J. Noronha-Hostler, N. Paladino, S. Rao, M.D. Sievert, D.E. Wertepny (2019), 1905.13323

[2] Y. Pandit (STAR), J. Phys. Conf. Ser. 458, 012003 (2013), 1305.0173

[3] H. Wang, P. Sorensen (STAR), Nucl. Phys. A932, 169 (2014), 1406.7522

[4] L. Adamczyk et al. (STAR), Phys. Rev. Lett. 115, 222301 (2015), 1505.07812

[5] D. Kharzeev, M. Nardi, Phys. Lett. B507, 121 (2001), nucl-th/0012025

[6] A. Goldschmidt, Z. Qiu, C. Shen, U. Heinz, Phys. Rev. C92, 044903 (2015), 1507.03910

[7] B. Schenke, C. Shen, P. Tribedy (2019), 1901.04378

[8] G. Giacalone, J. Noronha-Hostler, M. Luzum, J.Y. Ollitrault, Phys. Rev. C97, 034904 (2018), 1711.08499

[9] C. Collaboration (CMS) (2018)

[10] S. Acharya et al. (ALICE), Phys. Lett. B784, 82 (2018), 1805.01832

[11] T.A. collaboration (ATLAS), Measurement of the azimuthal anisotropy of charged particle production in Xe+Xe collisions at $\sqrt{s_{\mathrm{NN}}}=5.44 \mathrm{TeV}$ with the ATLAS detector, CERN (CERN, Geneva, 2018)

[12] C. Aidala et al. (PHENIX), Nature Phys. 15, 214 (2019), 1805.02973

[13] P. Bozek, W. Broniowski, Phys. Rev. Lett. 121, 202301 (2018), 1808. 09840

[14] M.D. Sievert, J. Noronha-Hostler, Phys. Rev. C100, 024904 (2019), 1901.01319

[15] M. Luzum, H. Petersen, J. Phys. G41, 063102 (2014), 1312. 5503

[16] J. Noronha-Hostler, L. Yan, F.G. Gardim, J.Y. Ollitrault, Phys. Rev. C93, 014909 (2016), 1511.03896

[17] J.S. Moreland, J.E. Bernhard, S.A. Bass, Phys. Rev. C92, 011901 (2015), 1412 . 4708

[18] Y.V. Kovchegov, D.E. Wertepny, Nucl.Phys. A906, 50 (2013), 1212. 1195

[19] Y.V. Kovchegov, D.E. Wertepny, Nucl. Phys. A925, 254 (2014), 1310.6701

[20] Y.V. Kovchegov, V.V. Skokov, Phys. Rev. D97, 094021 (2018), 1802.08166

[21] J.E. Bernhard, J.S. Moreland, S.A. Bass, J. Liu, U. Heinz, Phys. Rev. C94, 024907 (2016), 1605.03954 